\title{
Reachability Analysis of Linear Systems with Uncertain Parameters and Inputs
}

\author{
Matthias Althoff, Olaf Stursberg, and Martin Buss
}

\begin{abstract}
For a dynamic system with given initial state set, the reachable state set contains the states along all possible trajectories defined over (in-)finite time. This paper presents a method for computing conservative approximations of reachable sets for linear systems with uncertain system matrices and bounded inputs. Over- and underapproximations are computed for exponentials of system matrices with entries specified as bounded intervals. It is shown that reachable sets represented by sequences of zonotopes can be computed up to a dimension of 100 within a few seconds.
\end{abstract}

\section{INTRODUCTION}

The computation of reachable sets is of great interest for the safety verification of dynamic systems. Especially in the hybrid systems community, reachability computations are used to determine if a system can reach hazardous states, e.g. a robot arm should not collide with an obstacle or a liquid should not reach a boiling temperature. Hybrid systems unify discrete and continuous dynamics resulting in discrete and continuous reachable sets. In general, the computation of continuous reachable sets is more challenging than the discrete case, see e.g. [1]. For this reason, we exclusively deal with continuous reachability in this paper. Note that safety cannot be guaranteed by numeric simulation techniques if the initial state or the input is uncertain. Each simulation run provides a single trajectory for a single initial state and input trajectory. In case, the initial state or input is allowed to be taken from a subset of the continuous state or input space, the system can evolve in infinitely many ways. As one cannot check all possible trajectories, simulation techniques can only show that a system is unsafe if a single trajectory enters a dangerous state.

It has been shown that reachability analysis is decidable only for a limited class of systems [2]. Exemplary for this class are systems in which the derivatives of the continuous variables are specified as bounded intervals [3], [4], so that $\underline{a} \leq \dot{x} \leq \bar{a}$ with $x, \underline{a}, \bar{a} \in \mathbb{R}^{n}$. For other systems, such as systems with affine linear continuous dynamics where $\dot{x}=A x+b, x, b \in \mathbb{R}^{n}, A \in \mathbb{R}^{n \times n}$ reachability has to be determined in an approximative manner unless $A$ is of special structure [2]. Nevertheless, the avoidance of dangerous states concludes safety if the reachable set is overapproximated, see e.g. [5], [6]. This is because the avoidance of dangerous states for the overapproximated

All authors are with Institute of Automatic Control Engineering (LSR) , Technische Universität München, 80290 München, Germany $\{$ althoff, stursberg,mb\}@tum.de reachable set also holds for the exact solution, which is enclosed. A further extension to affine linear systems is the inclusion of uncertain inputs: $\dot{x} \in A x+v(t), v \in V$, $x, v \in \mathbb{R}^{n}, A \in \mathbb{R}^{n \times n}$ and $V$ is a convex set in $\mathbb{R}^{n}$. This is no specialization of the standard form $\dot{x}=A x+B u+v$ for linear systems as any input value $u$ and disturbance $v$ is considered by choosing $V=\left\{B u+v \mid u \in U, v \in V^{*}\right\}$. Reachability of those systems has been investigated, e.g. in [7], [8], [9]. Another class of systems that has been investigated in a series of three papers [10], [11], [12] are linear systems $\dot{x}=A x$, where $A$ is an interval matrix $A \in \mathcal{I}^{n \times n}$ when $\mathcal{I}$ is the set of all intervals $[c, d]$ with $c, d \in \mathbb{R}$ and $c \leq d$. This paper series showed reachability results for a single independent parameter $\theta \in \mathcal{I}$ resulting in a system matrix $A=G_{1}+\theta G_{2}, G_{1}, G_{2} \in \mathbb{R}^{n \times n}$ at certain time points without input. However, to the best knowledge of the authors, there is no comparable work to this one that is dealing with linear systems where all elements of $A$ are independent intervals. Additionally, in this paper reachability is not limited to the case of discrete time, but continuous time is used. Another distinction to the previous work of [10], [11], [12] is that time varying inputs are considered.

The content of this paper is presented in the following order: After a problem statement, some preliminaries on computation with interval matrices are introduced (Sec. III). Next, a scheme for computing over- and underapproximations of the exponential of interval matrices is described (Sec. IV). The interval matrix exponential is used to compute the reachable set of the linear system for the absence of inputs (Sec. (V)). The uncertain input is considered by translating and enlarging the reachable set of the homogeneous solution (Sec. VI). The methods are illustrated by two examples and the computational effort is discussed in terms of the system dimension.

\section{Problem Statement}

The objective is to compute the reachable set of a linear system with uncertain initial states, system matrices, and inputs. The initial state $x(0)$ can take values in a set $X_{0} \subset$ $\mathbb{R}^{n}$ and the input in a set $V \subset \mathbb{R}^{n}$. The evolution of the state $x$ is defined by the following differential equation:

$$
\dot{x}=A(t) x+v(t), \quad x(0) \in X_{0} \subset \mathbb{R}^{n}, \quad v(t) \in V \subset \mathbb{R}^{n}
$$

The system matrix is $A(t) \in \mathcal{A} \in \mathcal{I}^{n \times n}$, the elements of which are independent intervals of real numbers. Further, 
the system interval matrix is written as $\mathcal{A}=\langle\underline{A}, \bar{A}\rangle$ where $\underline{A}, \bar{A} \in \mathbb{R}^{n \times n}$ are the matrices determining the left and right limits of $\mathcal{A}$, so that one has for each element of $\mathcal{A}: \underline{a}_{i j} \leq$ $\bar{a}_{i j}, \forall i, j=1 . . n$, with $i$ denoting the row and $j$ the column of $\mathcal{A}$. The overapproximated set of reachable states of (1) in a time interval $t \in[0, r]$ is denoted by $R([0, r])$ and is defined over an auxiliary set $R(r)$ :

Definition 1: $R(r)$ is an overapproximation of the exact reachable set $R^{e}(r)$ that can be reached starting from $X_{0}$ (for $t=0$ ) at time $t=r$ :

$R^{e}(r)=\left\{x \mid x(t)\right.$ is a solution of (1), $\left.t=r, x(0) \in X_{0}\right\}$ and $R(r) \supseteq R^{e}(r)$.

Definition 2: $R([0, r])$ is the union of reachable sets $R(t)$ for $t \in[0, r]: R([0, r])=\bigcup_{t \in[0, r]} R(t)$

\section{PReliminaries}

A brief introduction to interval analysis is given and the consequences for interval matrix multiplications are discussed.

\section{A. Overapproximation in Scalar Interval Computations}

Basic results of interval analysis according to [13] are: Given are two intervals $a=[\underline{a}, \bar{a}] \in \mathcal{I}$ and $b=[\underline{b}, \bar{b}] \in \mathcal{I}$, the addition and multiplication of two intervals can be obtained as

$$
\begin{aligned}
a+b & =[\underline{a}+\underline{b}, \bar{a}+\bar{b}] \\
a b & =[\min (\underline{a b}, \underline{a} \bar{b}, \bar{a} \underline{b}, \bar{a} \bar{b}), \max (\underline{a b}, \underline{a} \bar{b}, \bar{a} \underline{b}, \bar{a} \bar{b})]
\end{aligned}
$$

With (2), one can compute the range of a function such as $c=a b+a$ where e.g. $a=[-2,-1]$ and $b=[-1,1]$. Applying interval arithmetics, the computation of $c$ can be performed in two ways:

$$
c=a b+a=[-4,1], \quad c=a(b+1)=[-4,0]
$$

Although interval arithmetics can guarantee that the exact solution is always included, only the second computation gives the exact solution. Exact results can be guaranteed if each variable occurs only once in interval computations such as in the second expression of $c$. In consistency to [14], these expressions are referred to as single-use expressions (SUE). In the other case, one generally receives an overapproximation as each variable occurring in different interval computations is allowed to have different values. In literature this problem is also referred to as the dependency problem [13].

\section{B. Exactness of Interval Matrix Multiplication}

The multiplication of interval matrices has been investigated in [14] recently. The results are briefly presented in this subsection and differ substantially if two or three and more matrices are multiplied: two matrices are multiplied to $C=M N$ with $M \in \mathcal{I}^{o \times p}$ and $N \in \mathcal{I}^{p \times q}$ by computing the elements of $C$ as $c_{i j}=\sum_{k=1}^{p} m_{i k} n_{k j}, \forall i=1 . . o, j=1 . . q$ using standard interval arithmetics as introduced in (2). As the expression of $c_{i j}$ is a SUE, interval arithmetics returns the exact result. It is also shown that one can reformulate $P^{2}$, $P \in \mathcal{I}^{s \times s}$ as a SUE, which also allows an exact computation using interval arithmetics. In contrast, the multiplication of three matrices $D=M N Q$ with $M \in \mathcal{I}^{o \times p}, N \in \mathcal{I}^{p \times q}$ and $Q \in \mathcal{I}^{q \times w}$ cannot be computed exactly with interval arithmetics. It is also proven that the exact computation with other methods is NP-hard. The same result holds for a cubic interval matrix $P^{3}, P \in \mathcal{I}^{s \times s}$. Additionally, matrix multiplication is not associative when using interval arithmetics: $(A A) A \neq A(A A), A \in \mathcal{I}^{n \times n}$. For consistency reasons, matrix multiplication is always performed from 'left to right' in this paper: $((A A) . . A)$.

\section{INTERVAL MATRIX EXPONENTIAL}

Inner and outer bounds of the matrix exponential $e^{\mathcal{A} t}$ are computed, where $\mathcal{A} \in \mathcal{I}^{n \times n}$ is an interval matrix and $t \in$ $\mathbb{R}^{+}$. The matrix exponential is obtained by a Taylor series, see [15]:

$$
e^{\mathcal{A} t}=I+\mathcal{A} t+\frac{1}{2 !}(\mathcal{A} t)^{2}+\frac{1}{3 !}(\mathcal{A} t)^{3}+\ldots
$$

$I \in \mathbb{R}^{n \times n}$ is the identity matrix. In order to obtain $e^{\mathcal{A} t}$ in a finite number of steps, the Taylor series is cut off after $p$ terms, which is denoted $e_{p}^{\mathcal{A} t}$. An over- as well as an underapproximation of $e_{p}^{\mathcal{A} t}$ is computed using the following lemma which follows the principle of the exact computation of the square of an interval matrix in [14]:

Lemma 1 (Exact computation of $\mathcal{A} t+\frac{1}{2} \mathcal{A}^{2} t^{2}$ ): The expression $W(t)=\mathcal{A} t+\frac{1}{2} \mathcal{A}^{2} t^{2}$ can be computed exactly by the following procedure using interval arithmetics:

$$
\begin{aligned}
\forall i \neq j: w_{i j} & =a_{i j}\left(t+\frac{1}{2}\left(a_{i i}+a_{j j}\right) t^{2}\right) \\
& +\frac{1}{2} \sum_{k: k \neq i, k \neq j} a_{i k} a_{k j} t^{2} \\
\forall i: w_{i i}= & \left.\kappa\left(a_{i i}, t\right), \max \left(\left\{\underline{a}_{i i} t+\frac{1}{2} \underline{a}_{i i}^{2} t^{2}, \bar{a}_{i i} t+\frac{1}{2} \bar{a}_{i i}^{2} t^{2}\right\}\right)\right] \\
& +\frac{1}{2} \sum_{k: k \neq i} a_{i k} a_{k i} t^{2} \\
\kappa\left(a_{i i}, t\right)= & \left\{\begin{array}{l}
\min \left(\left\{\underline{a}_{i i} t+\frac{1}{2} \underline{a}_{i i}^{2} t^{2}, \bar{a}_{i i} t+\frac{1}{2} \bar{a}_{i i}^{2} t^{2}\right\}\right),-\frac{1}{t} \notin a_{i i} \\
-\frac{1}{2}, \quad-\frac{1}{t} \in a_{i i}
\end{array}\right.
\end{aligned}
$$

Proof: As one can reformulate the computation of $w_{i j}$ into a SUE as presented in (3), the computation of $w_{i j}$ with interval arithmetics is exact. The computation of $w_{i i}$ cannot be reformulated to a SUE. However, one can split $w_{i i}$ into a part with and without a single variable occurrence: $w_{i i}=$ $a_{i i} t+\frac{1}{2} a_{i i}^{2} t^{2}+\frac{1}{2} \sum_{k: k \neq i} a_{i k} a_{k i} t^{2}$. As $\frac{1}{2} \sum_{k: k \neq i} a_{i k} a_{k i} t^{2}$ is a SUE, it remains to compute the minimum and maximum of $\gamma\left(a_{i i}\right):=a_{i i} t+\frac{1}{2} a_{i i}^{2} t^{2}$. The function $\gamma\left(a_{i i}\right)$ has only one minimum at $a_{i i}=-\frac{1}{t}$ and is monotone elsewhere, so that one can compute $\gamma_{\max }=\max \left(\left\{\underline{a}_{i i} t+\frac{1}{2} \underline{a}_{i i}^{2} t^{2}, \bar{a}_{i i} t+\frac{1}{2} \bar{a}_{i i}^{2} t^{2}\right\}\right)$. In case the global minimum $\left(a_{\min }=-\frac{1}{t}\right)$ is an element of $a_{i i}$, one gets: $\gamma_{\min }=-\frac{t}{t}+\frac{1}{2} \frac{t^{2}}{t^{2}}=-\frac{1}{2}$. In the other case, the minimum is computed as $\gamma_{\min }=\min \left(\left\{\underline{a}_{i i} t+\frac{1}{2} \underline{a}_{i i}^{2} t^{2}, \bar{a}_{i i} t+\right.\right.$ $\left.\left.\frac{1}{2} \bar{a}_{i i}^{2} t^{2}\right\}\right)$. 
After defining $1 \in \mathbb{R}^{n \times n}$ as a matrix of ones, the interval matrix exponential $e^{A t}$ is overapproximated by the following theorem.

Theorem 1 (Overapproximation of $\left.e^{\mathcal{A t}}\right)$ : The overapproximation of the interval matrix exponential with order $p$, denoted $\left\lceil e_{p}^{\mathcal{A} t}\right\rceil$, where $\mathcal{A} \in \mathcal{I}^{n \times n}$ and $t \in \mathbb{R}^{+}$ is obtained by

$$
\begin{aligned}
\left\lceil e_{p}^{\mathcal{A} t}\right\rceil & =I+W(t)+\sum_{i=3}^{p} \frac{1}{i !}(\mathcal{A} t)^{i}+E(t), \\
E(t) & =\langle-\mathbf{1}, \mathbf{1}\rangle \frac{\left(\|\mathcal{A}\|_{\infty} t\right)^{p+1}}{(p+1) !} \frac{1}{1-\epsilon}, \quad \epsilon=\frac{\|\mathcal{A}\|_{\infty} t}{p+2} \stackrel{!}{<} 1
\end{aligned}
$$

Proof: The interval matrix exponential is computed by a finite Taylor expansion. The first part $I+W$ of the Taylor expansion is exact, as $I$ is not an interval matrix and the exactness of $W=\mathcal{A} t+\frac{1}{2} \mathcal{A}^{2} t^{2}$ has been shown in lemma 1. The next sum of the Taylor expression up to order $p$ is computed by interval arithmetics, which returns overapproximations. As the Taylor expansion is finite, it is necessary to give an overapproximation for the remainder $E$, which has been derived in [16]. Note, that for the computation of $E$, it is required to choose $p$ so that $\epsilon<1$ as $\frac{1}{1-\epsilon}$ is obtained from $1+\epsilon+\epsilon^{2}+\ldots$ in [16].

The difference to the computation of $E$ for a fixed matrix is that $\|\mathcal{A}\|_{\infty}$ has to be computed for an interval matrix:

$$
\|\mathcal{A}\|_{\infty}=\|\max (|\underline{A}|,|\bar{A}|)\|_{\infty}
$$

The absolute value and the maximum in the above expression are determined elementwise. In order to obtain an interval for the exact solution of the interval matrix exponential, the underapproximation is presented in the following theorem:

Theorem 2 (Underapproximation of $e^{\mathcal{A} t}$ ): An underapproximation of the interval matrix exponential, denoted $\left\lfloor e_{p}^{\mathcal{A t}}\right\rfloor$ can be computed as

$$
\begin{aligned}
\left\lfloor e_{p}^{\mathcal{A} t}\right\rfloor= & I+W(t) \\
& +\langle\min (\{Y(t), Z(t)\}), \max (\{Y(t), Z(t)\})\rangle \\
Y(t)= & \sum_{i=3}^{p} \frac{1}{i !} \underline{A}^{i} t^{i}, \quad Z(t)=\sum_{i=3}^{p} \frac{1}{i !} \bar{A}^{i} t^{i}
\end{aligned}
$$

Proof: Analog to the overapproximation, the underapproximation is computed based on the Taylor series of the matrix exponential. Lemma 1 states that the first part of the Taylor expansion is exact. The next expression $\langle\min (\{Y(t), Z(t)\}), \max (\{Y(t), Z(t)\})\rangle$ returns the interval of solutions for the Taylor polynomials of order 3 to $p$ that is spanned by the sample matrices $\underline{A}$ and $\bar{A}$. As $\underline{A}, \bar{A} \in A$, it follows that $Y(t), Z(t) \in \sum_{i=3}^{p} \frac{1}{i !} \mathcal{A}^{i} t^{i}$. As the matrix exponential is a continuous function, all solutions in between $Y(t)$ and $Z(t)$ are a solution that underapproximate the exact solution.

The quality of the over- and underapproximation is illustrated by a simple example.
Example 1: Given is the following interval matrix $\mathcal{A}$ :

$$
\mathcal{A}=\left[\begin{array}{cc}
{[-1.1,-0.9]} & {[-4.1,-3.9]} \\
{[3.9,4.1]} & {[-1.1,-0.9]}
\end{array}\right], \quad t=0.04
$$

The results of $\left\lfloor e_{4}^{\mathcal{A} t}\right\rfloor$ and $\left\lceil e_{4}^{\mathcal{A} t}\right\rceil(p=4)$ are

$$
\begin{aligned}
\left\lfloor e_{4}^{\mathcal{A} t}\right\rfloor & =\left[\begin{array}{lc}
{[0.94408,0.95295]} & {[-0.15758,-0.14859]} \\
{[0.14865,0.15753]} & {[0.94408,0.95295]}
\end{array}\right] \\
\left\lceil e_{4}^{\mathcal{A} t}\right\rceil & =\left[\begin{array}{lc}
{[0.94396,0.95309]} & {[-0.15765,-0.14852]} \\
{[0.14852,0.15765]} & {[0.94396,0.95309]}
\end{array}\right]
\end{aligned}
$$

The overapproximation of the interval matrix exponential is used in the next section to compute the reachable set of the uncertain linear system (1) without input.

\section{REACHABILITY WITHOUT INPUT}

The reachable set without input, denoted $\hat{R}([0, r])$, is computed by steps that are similar to other approaches, e.g. [17], [7], [5], [18], but extended to interval computations: First, the reachable set $\hat{R}(r)$ at the time point $t=r$ is computed. Next, the convex hull of the reachable set $\hat{R}(r)$ and the set of initial states $X_{0}$ is calculated. This convex hull is enlarged to $\hat{R}([0, r])$ by a certain amount, ensuring the enclosure of the reachable set for all times of the interval $t \in[0, r]$. The computation of the enlarging amount differs from other approaches. The basic steps for the computation of $\hat{R}([0, r])$ are illustrated in Fig. 1.

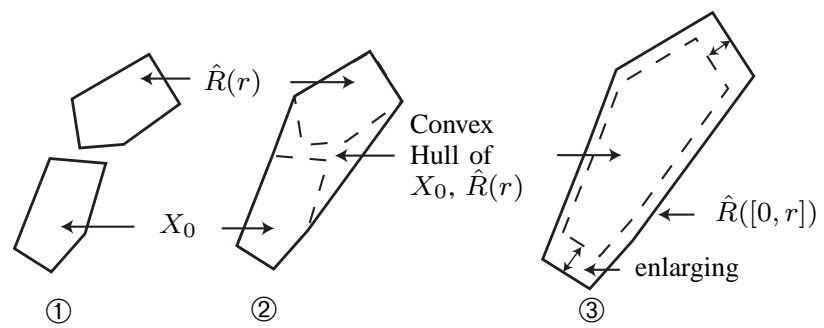

Fig. 1. Computation of Reachable Sets - Overview

\section{A. Reachable set starting from a single point}

The solution of (1) at time $t=r$ for a system without input $v(t)$, starting from a single point $x_{0}$ so that $x(0)=x_{0}$ is well known to be calculated by:

$$
x(r)=e^{\int_{0}^{r} A(t) d t} x(0)
$$

The integral is circumvented by the following proposition:

Proposition 1 (Replacing Integrals by Intervals): The following integral can be replaced by the interval of its integrand: $\int_{0}^{r} A(t) d t \in \mathcal{A} r, A(t) \in \mathcal{A}$.

Proof: The integral is written as a Riemann integral:

$$
\begin{aligned}
\int_{0}^{r} A(t) d t & =\lim _{n \rightarrow \infty} \sum_{i=1}^{n} A\left(t_{i}\right)\left(t_{i}-t_{i-1}\right), 0 \leq t_{i-1}<t_{i} \leq r \\
& \in \lim _{n \rightarrow \infty} \sum_{i=1}^{n} \mathcal{A}\left(t_{i}-t_{i-1}\right)=\mathcal{A} r
\end{aligned}
$$


Proposition 1 allows to reformulate (4):

$$
x(r) \in e^{\mathcal{A} r} x(0) \subseteq\left\lceil e_{p}^{\mathcal{A} t}\right\rceil x(0)
$$

and $\left\lceil e_{p}^{\mathcal{A} t}\right\rceil$ is computed as presented in theorem 1. In a next step, the solution for $t \in[0, r]$ is approximated by a convex hull of the solutions at time $t=r$ and $t=0$, see [7]. A state contained in the convex hull is given by:

$x(t) \in\left\lceil e_{p}^{\mathcal{A} t}\right\rceil x(0) \approx x(0)+\frac{t}{r}\left(\left\lceil e_{p}^{\mathcal{A} r}\right\rceil x(0)-x(0)\right), \quad t \in[0, r]$

After denoting an operator for computing the convex hull by $\mathcal{C H}()$, one can replace $x(0)+\frac{[0, r]}{r}\left(\left\lceil e_{p}^{\mathcal{A} r}\right\rceil x(0)-x(0)\right)$ by $\mathcal{C H}\left(x(0),\left\lceil e_{p}^{\mathcal{A} r}\right\rceil x(0)\right)$. In order to ensure that the solution of $x(t)$ is enclosed by the convex hull for any $t \in[0, r]$, it is enlarged by a Minkowski addition of a correction term $F x(0)$ :

$$
\begin{aligned}
\left\lceil e_{p}^{\mathcal{A} t}\right\rceil x(0) & \subseteq x(0)+\frac{t}{r}\left(\left\lceil e_{p}^{\mathcal{A} r}\right\rceil x(0)-x(0)\right)+F x(0) \\
\Rightarrow F & \stackrel{!}{\supseteq}\left\lceil e_{p}^{\mathcal{A} t}\right\rceil-I-\frac{t}{r}\left(\left\lceil e_{p}^{\mathcal{A} r}\right\rceil-I\right), \quad \forall t \in[0, r]
\end{aligned}
$$

so that

$$
\hat{R}([0, r])=\mathcal{C H}\left(x(0),\left\lceil e_{p}^{\mathcal{A} r}\right\rceil x(0)\right)+F x(0)
$$

The computation of $F$ is discussed in the following theorem.

Theorem 3 (Correction hull $F$ ): The set $F$ that is added to the convex hull of time point solutions at $t=0$ and $t=r$ to ensure enclosure of all solutions between these times, can be computed by:

$$
F=\sum_{i=2}^{p}\left[\left(i^{\frac{-i}{i-1}}-i^{\frac{-1}{i-1}}\right) r^{i}, 0\right] \frac{\mathcal{A}^{i}}{i !}+E(r)
$$

with an interval system matrix $\mathcal{A}$ and an interval matrix exponential remainder $E(r)$ according to theorem 1 .

Proof: The value of $F$ is calculated from (5) as:

$$
\begin{aligned}
\left\lceil e_{p}^{\mathcal{A} t}\right\rceil-I-\frac{t}{r}\left(\left\lceil e_{p}^{\mathcal{A} r}\right\rceil-I\right) & =\sum_{i=2}^{p}\left(t^{i}-t r^{i-1}\right) \frac{1}{i !} \mathcal{A}^{i} \\
& +E(t)-\frac{t}{r} E(r)
\end{aligned}
$$

Note, that the first order term of $\left\lceil e_{p}^{\mathcal{A} t}\right\rceil$ and $\left\lceil e_{p}^{\mathcal{A} r}\right\rceil$ cancel out. Next, bounds on $t^{i}-t r^{i-1}, t \in[0, r]$ are given. As $t^{i}-t r^{i-1}=0$ for $t=0, t=r$, and since there is only one minimum on the interval $t \in[0, r]$, one obtains:

$$
\begin{aligned}
& \frac{d}{d t}\left(t^{i}-t r^{i-1}\right) \stackrel{!}{=} 0 \rightarrow t_{\min }=i^{-\frac{1}{i-1}} r, \quad t \in[0, r] \\
& \quad \rightarrow t^{i}-t r^{i-1} \in\left[\left(i^{\frac{-i}{i-1}}-i^{\frac{-1}{i-1}}\right) r^{i}, 0\right], \quad \forall t \in[0, r]
\end{aligned}
$$

It remains to compute bounds for the interval matrix exponential remainder $E$ :

$$
\begin{gathered}
E(t)=\langle-\mathbf{1}, \mathbf{1}\rangle \phi(t), \quad \phi(t)=\frac{\left(\|\mathcal{A}\|_{\infty} t\right)^{p+1}}{(p+1) !} \frac{1}{1-\epsilon} \\
t \in[0, r]: E(t)-\frac{t}{r} E(r)=\langle-\mathbf{1}, \mathbf{1}\rangle(\underbrace{\phi(t)}_{\geq 0}-\underbrace{t / r}_{\leq 1} \phi(r)) \\
\subset\langle-\mathbf{1}, \mathbf{1}\rangle \phi(r)=E(r)
\end{gathered}
$$

\section{B. Reachable set starting from a zonotope}

Based on the computation of the reachable set of a single initial value, the reachable set starting from a zonotope [7] is discussed in this section. A zonotope $\mathrm{Z}$ is a set such that:

$$
Z=\left\{x \in \mathbb{R}^{n}: x=c+\sum_{i=1}^{q}[-1,1] g^{(i)}\right\}
$$

where $c \in \mathbb{R}^{n}$ is the center and $g^{(1)}, \ldots, g^{(q)} \in \mathbb{R}^{n}$ are generators that are indexed by raised numbers in parenthesis. Zonotopes are denoted as $Z=\left(c, g^{(1)}, \ldots, g^{(q)}\right)$ and the order of a zonotope is $\frac{q}{n}$. Zonotopes are used for the computation of reachable sets for three reasons: First, they are closed under linear transformation, see [7]. Let $\mathcal{L}$ be a linear map that can be described by a matrix $L$, the mapped zonotope is:

$$
\begin{aligned}
\mathcal{L}(Z) & =\left\{x \in \mathbb{R}^{n}: x=L c+\sum_{i=1}^{q}[-1,1] L g^{(i)}\right\} \\
& =\left(L c, L g^{(1)}, \ldots, L g^{(q)}\right)
\end{aligned}
$$

In the remaining text, $L Z$ is written instead of $\mathcal{L}(Z)$. Second, zonotopes are closed under Minkowski sum. This is very helpful when the reachable set has to be enlarged due to the error term for time interval computations or due to uncertain inputs. The Minkowski sum $Z_{1}+Z_{2}$ of two zonotopes $Z_{1}=\left(c_{1}, g^{(1)}, \ldots, g^{(q)}\right)$ and $Z_{2}=\left(c_{2}, h^{(1)}, \ldots, h^{(u)}\right)$ is, as shown in [7]:

$$
Z_{1}+Z_{2}=\left(c_{1}+c_{2}, g^{(1)}, \ldots, g^{(q)}, h^{(1)}, \ldots, h^{(u)}\right)
$$

Third, an overapproximation of the map of an interval matrix with a zonotope can be obtained in a simple manner, as shown in the following:

Lemma 2 (Symmetric Interval Matrix Multiplication): The multiplication of a matrix $T \in \mathbb{R}^{n \times n}$ with a symmetric interval matrix $S=\langle-\hat{S}, \hat{S}\rangle \in \mathcal{I}^{n \times n}$ can be computed exactly as follows:

$$
T S=\langle-|T| \hat{S},|T| \hat{S}\rangle, \quad S T=\langle-\hat{S}|T|, \hat{S}|T|\rangle
$$

The absolute value is applied elementwise. If $S \in \mathcal{I}$, the computation of (6) results in an overapproximation.

Proof: Without loss of generality, the multiplication with $S=\langle-\hat{S}, \hat{S}\rangle \in \mathcal{I}^{n \times n}$ is shown for the multiplication of $t \in \mathbb{R}^{1 \times n}$ (a row vector of $T$ ) and $s=\langle-\hat{s}, \hat{s}\rangle \in$ $\mathcal{I}^{n \times 1}$ (a column vector of $S$ ). The maximum value of $t s$ is obtained by selecting $s_{i}=\operatorname{sign}\left(t_{i}\right) \hat{s}_{i}, \forall i=1 . . n$ so that $\max (t s)=\sum_{i=1}^{n}|t|_{i} \hat{s}_{i}$. Analogously, the minimum is obtained by choosing $s_{i}=-\operatorname{sign}\left(t_{i}\right) s_{i}, \forall i=1 . . n$ so that $\min (t s)=-\sum_{i=1}^{n}|t|_{i} \hat{s}_{i}$. These computations are exact as they are SUE. If $S \in \mathcal{I}$, the computations are not SUE anymore, as the same interval variable $S$ has to be used for each multiplication with an element of $T$. Thus, (6) is an overapproximation if $S \in \mathcal{I}$.

Lemma 3 (Symmetric Interval Matrix map for Zonotopes): The linear map of a symmetric interval matrix $N \in\langle-\hat{N}, \hat{N}\rangle$ 
with a zonotope is overapproximated by a hyperrectangle with center 0 :

$$
\begin{aligned}
N Z & =\left\{x \in \mathbb{R}^{n}: x=\langle-\hat{N}, \hat{N}\rangle c+\sum_{i=1}^{q}[-1,1]\langle-\hat{N}, \hat{N}\rangle g^{(i)}\right\} \\
& =\left(0, v^{(1)}, \ldots, v^{(n)}\right) \\
v_{j}^{(i)} & = \begin{cases}0, & i \neq j \\
\hat{N}_{j}\left(|c|+\sum_{k=1}^{q}|g|^{(k)}\right), \quad i=j\end{cases}
\end{aligned}
$$

and the subscript $j$ of $v_{j}^{(i)}$ denotes the $j^{\text {th }}$ element of $v^{(i)}$ and $\hat{N}_{j}$ denotes the $j^{\text {th }}$ row of $\hat{N}$.

Proof: Using lemma 2 with $S=[-1,1]$ and $T=$ $g^{(k)}$, the zonotope $Z=c+\sum_{k=1}^{q}[-1,1] g^{(k)}$ is overapproximated by $Z=c+\sum_{k=1}^{q}|g|^{(k)}$ as $S \in \mathcal{I}$. The multiplication with $N$ yields under application of lemma 2: $N Z=\left\langle-\hat{N}\left(|c|+\sum_{k=1}^{q}|g|^{(k)}\right), \hat{N}\left(|c|+\sum_{k=1}^{q}|g|^{(k)}\right)\right\rangle=$ $\sum_{i=1}^{n}[-1,1] v^{(i)}$ where $v^{(i)}$ is defined as in lemma 3 .

An examplary illustration of the overapproximation defined by lemma 3 is shown in Fig. 2(a) for $N=$ $\operatorname{diag}([-1,1],[-1,1])$. Lemma 3 allows to formulate an overapproximation for the linear map of a zonotope $Z$ with an interval matrix $M$ :

Theorem 4 (Interval Matrix map for Zonotopes): The linear map of a zonotope $Z=\left(c, g^{(1)}, \ldots, g^{(q)}\right)$ represented by an interval matrix $M=\hat{M}+\langle-\hat{M}, \hat{M}\rangle$ where $\tilde{M}, \hat{M} \in \mathbb{R}^{n \times n}$ is overapproximated by:

$$
\begin{aligned}
M Z & =\left(\tilde{M} c, \tilde{M} g^{(1)}, \ldots, \tilde{M} g^{(q)}, v^{(1)}, \ldots, v^{(n)}\right) \\
v_{j}^{(i)} & =\left\{\begin{array}{l}
0, \quad i \neq j \\
\hat{M}_{j}\left(|c|+\sum_{k=1}^{q}|g|^{(k)}\right), \quad i=j
\end{array}\right.
\end{aligned}
$$

Proof: The interval matrix multiplication with $M$ is split up into a matrix multiplication with $\tilde{M}$ and a symmetric interval matrix multiplication with $\langle-\hat{M}, \hat{M}\rangle: M Z=$ $\tilde{M} Z+\langle-\hat{M}, \hat{M}\rangle Z$. The result of the matrix multiplication is denoted $L_{1}=\tilde{M} Z$. The linear map of the symmetric interval matrix obtained from lemma 3 is: $L_{2}=\langle-\hat{M}, \hat{M}\rangle Z=$ $\left(0, v^{(1)}, \ldots, v^{(n)}\right)$. Applying the Minkowski addition rule for $M Z=L_{1}+L_{2}$ yields the final result of theorem 4. An overapproximation occurs as the intervals $[-1,1]$ in $\tilde{M} Z$ and $\langle-\hat{M}, \hat{M}\rangle Z$ for the same generator $g^{(i)}$ are treated as independent intervals although they are originally identical.

The overapproximation of theorem 4 is best illustrated for the multiplication with a generator that has only one generator $g^{(1)}$ as shown in Fig. 2(b). Note, that the exact reachable set is not a zonotope anymore.

Equivalent to the solution of the reachable set starting from a single point $x(0)$, the reachable set $\hat{R}([0, r])$ starting from a zonotope $Z_{0}$ without input $(v=0)$ can be computed as follows:

$$
\hat{R}([0, r])=\mathcal{C H}\left(Z_{0},\left\lceil e_{p}^{\mathcal{A} r}\right\rceil Z_{0}\right)+F Z_{0}
$$

The convex hull $\mathcal{C H}()$ of two zonotopes is a polytope in general. For this reason, a zonotope that encloses the convex

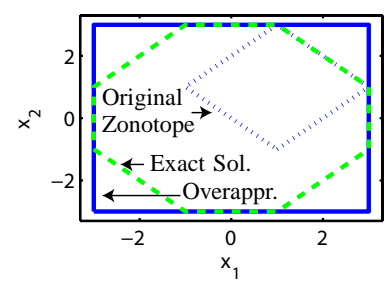

(a) Example for lemma 3

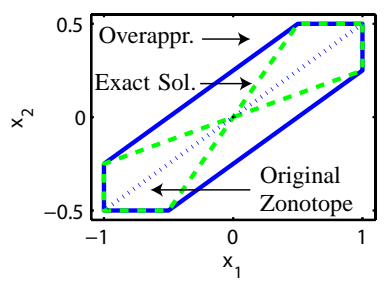

(b) Example for theorem 4

Fig. 2. Overapproximations occurring in lemma 3 and theorem 4

hull is constructed as presented in [7]. Due to the fact that $e^{\mathcal{A} r} e^{\mathcal{A} t}=e^{\mathcal{A}(r+t)}$, one can compute the reachable set for any interval $t \in[(k-1) r, k r], k \in \mathbb{N}^{+}$by:

$$
\hat{R}([k r,(k+1) r])=\left\lceil e_{p}^{\mathcal{A} k r}\right\rceil \hat{R}([0, r])
$$

The reachable set $\hat{R}([0, T])$ with $T=k r$ is easily obtained by unifying the reachable sets of computed subintervals:

$$
\hat{R}([0, T])=\bigcup_{k=1}^{T / r} \hat{R}([(k-1) r, k r])
$$

where a smaller value of $r$, which can be freely chosen, enhances the accuracy of $\hat{R}([0, T])$.

\section{REACHABILITY WITH INPUTS}

In this section, the reachable set $R([0, r])$ of the linear system (1) is computed for an input $v(t)$ defined on a zonotope V. Due to the linearity of the system, the reachable set is computed by superposition of the homogeneous and the particulate solution:

$$
R([0, r])=\hat{R}([0, r])+\bar{R}([0, r])
$$

While the solution of $\hat{R}([0, r])$ has been described already in the previous section, the reachable set of the particulate solution $\bar{R}([0, r])$ is now investigated. The particulate solution $x_{p}(r)$ of a linear system with time varying system matrix $A(t)$ and uncertain input $v(t) \in V$ is:

$$
x_{p}(r)=e^{\int_{0}^{r} A(t) d t} \int_{0}^{r} e^{\int_{0}^{t}-A(\tau) d \tau} v(t) d t
$$

Applying proposition 1 , one can simplify the particulate solution to

$$
x_{p}(r)=e^{\mathcal{A} r} \int_{0}^{r} e^{-\mathcal{A} t} v(t) d t=\int_{0}^{r} e^{\mathcal{A}(r-t)} v(t) d t
$$

Following the same principle as in proposition 1, one can overapproximate the particulate solution using the set of inputs $V$ :

$$
\begin{aligned}
x_{p}(r) & =\int_{0}^{r} e^{\mathcal{A}(r-t)} v(t) d t \\
& =\lim _{n \rightarrow \infty} \sum_{i=1}^{n} e^{\mathcal{A}\left(r-t_{i}\right)} v\left(t_{i}\right)\left(t_{i}-t_{i-1}\right), 0 \leq t_{i-1}<t_{i} \leq r \\
& \in \lim _{n \rightarrow \infty} \sum_{i=1}^{n} e^{\mathcal{A}\left(r-t_{i}\right)} V\left(t_{i}-t_{i-1}\right)=\int_{0}^{r} e^{\mathcal{A}(r-t)} d t V
\end{aligned}
$$


By substituting $\tau=r-t$, the particulate solution can be further simplified to

$$
x_{p}(r) \in \int_{0}^{r} e^{\mathcal{A}(r-t)} V d t=\int_{0}^{r} e^{\mathcal{A} \tau} V d \tau
$$

Applying theorem 1, one can state the following proposition:

Proposition 2 (Particulate Reachable Set): Using interval arithmetics and the exact value for $W^{*}(r)=$ $\frac{1}{2} A r^{2}+\frac{1}{6} A^{2} r^{3}$, the reachable set $\bar{R}([0, r])$ of the particulate solution can be overapproximated as:

$$
\begin{aligned}
\bar{R}([0, r])= & \left(I r+W^{*}(r)\right) V \\
+ & \sum_{i=3}^{p} \frac{1}{i !(i+1)} \mathcal{A}^{i} r^{i+1} V+E(r) r V
\end{aligned}
$$

Proof: All possible solutions of $x_{p}(r)$ in (7) can be overapproximated by substituting $e^{\mathcal{A} \tau}$ by $\left\lceil e_{p}^{\mathcal{A} \tau}\right\rceil$ :

$$
\begin{aligned}
\bar{R}([0, r])= & \left(I r+W^{*}(r)\right) V \\
& +\sum_{i=3}^{p} \frac{1}{i !} \mathcal{A}^{i} V \int_{0}^{r} \tau^{i} d \tau+\int_{0}^{r} E(\tau) d \tau V
\end{aligned}
$$

The value of $W^{*}=\frac{1}{2} \mathcal{A} r^{2}+\frac{1}{6} \mathcal{A}^{2} r^{3}$ is computed as presented in lemma 1 with some minor adjustments as $W^{*} \neq W$, i.e. $I r+W^{*}(r)$ is exact. The multiplications of $\left(I r+W^{*}(r)\right)$ and $\mathcal{A}^{n}$ with $V$ are performed as in theorem 4, such that the result is an overapproximation. The result of computing the matrix $\mathcal{A}^{i}$ is also an overapproximation as the expression is not a SUE. The time integrals result in $\int_{0}^{r} \tau^{i} d \tau=r^{i+1} /(i+$ $1)$. It remains to specify the following overapproximation: $\int_{0}^{r} E(\tau) d \tau \subset E(r) \int_{0}^{r} d \tau=E(r) r$ as $E(t)$ is monotone for $\epsilon<1$ and $t \in[0, r]$.

Finally, algorithm 1 for the computation of $R([0, T]), T=$ $k r$ is stated. Note that the order of the zonotopes $R([(k-$ 1) $r, k r])$ constantly grows with $k$ due to the computation of $\left\lceil e_{p}^{\mathcal{A} r}\right\rceil R([(k-2) r,(k-1) r])$ and the Minkowski addition of $\bar{R}([0, r])$. For this reason, order reduction techniques are used as presented in [7] which allow to not exceed a maximum order of the zonotopes.

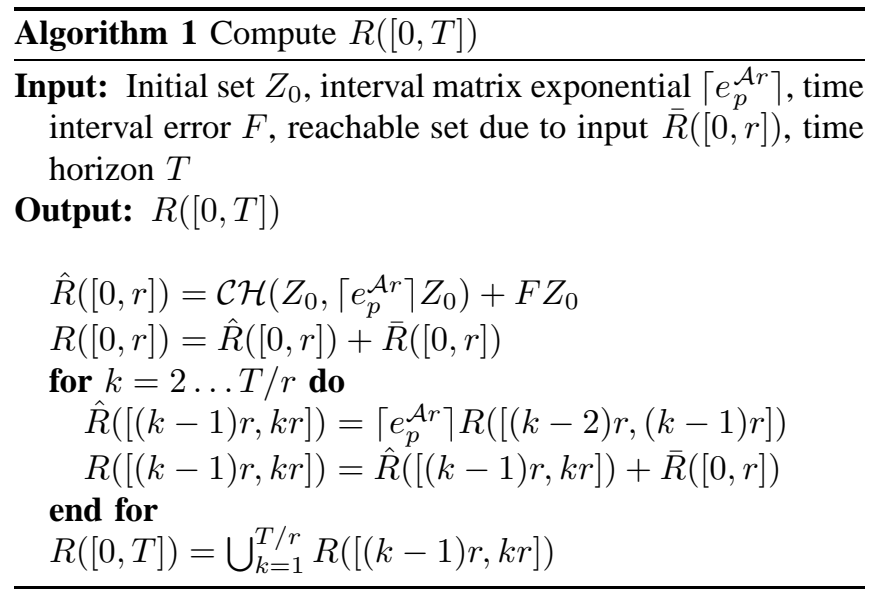

\section{EXAMPLES}

First, a simple two dimensional example is presented which is taken from [7] and extended by parameter uncertainties with the following specifications: Initial values are within $[0.9,1.1]$ for each dimension. The time step is chosen as $r=0.04$ for a time interval $t \in[0,5](125$ iterations), and Taylor expansions of order 4 are used to compute $\left\lceil e_{p}^{\mathcal{A} r}\right\rceil, F$ and $\bar{R}([0, r])$. The maximum zonotope order is 10. All interval computations are performed with the tool $b 4 m$ [19]. The model of the first example is given as follows:

$\dot{x}=\left[\begin{array}{cc}{[-1.05,-0.95]} & {[-4.05,-3.95]} \\ {[3.95,4.05]} & {[-1.05,-0.95]}\end{array}\right] x+\left[\begin{array}{l}1 \\ 1\end{array}\right][-0.05,0.05]$

Its overapproximated reachable set together with sample trajectories starting in the vertices of $Z_{0}$ are shown for three cases in Fig. 3. The sample trajectories bound the true reachable area and the zonotopes represent the overapproximated reachable set. The first case shows the reachable set without input. The second one is computed without uncertain parameters: $A^{*}=\underline{A}+0.5(\bar{A}-\underline{A})$. The third one shows the reachable sets with uncertain parameters and input.

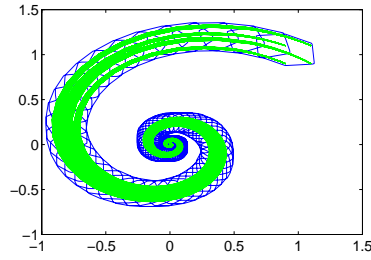

(a) No input

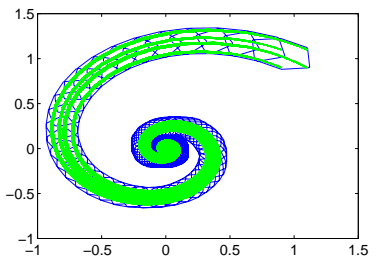

(b) No uncertain parameters

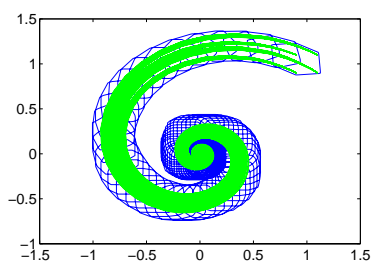

(c) Uncertain parameters and input

Fig. 3. Reachable sets for the 2 dimensional example

The second example is also taken from [7] - but it is extended to an uncertain system matrix, the disturbance input is specified as a zonotope, and a modification is chosen in which the dynamics of $x_{2}$ and $x_{3}$ is coupled. The specifications are the same as for the first example, except that the maximum order of the zonotopes is 5 . The model of the second example is given as follows:

$$
\begin{aligned}
\dot{x} & =(\tilde{A}+\langle-\hat{A}, \hat{A}\rangle) x+V \\
\tilde{A} & =\left[\begin{array}{ccccc}
-1 & -4 & 0 & 0 & 0 \\
4 & -1 & 1 & 0 & 0 \\
0 & 0 & -3 & 1 & 0 \\
0 & 0 & -1 & -3 & 0 \\
0 & 0 & 0 & 0 & -2
\end{array}\right]
\end{aligned}
$$


TABLE I

COMPUTATIONAL COMPLEXITY

\begin{tabular}{|l||c|c|c|c|c|}
\hline Dimension $n$ & 5 & 10 & 20 & 50 & 100 \\
\hline $\begin{array}{l}\text { CPU-time [sec] } \\
\left(\left\lceil e_{p}^{\mathcal{A} r}\right\rceil, F, \bar{R}([0, r])\right)\end{array}$ & 0.04 & 0.09 & 0.22 & 1.15 & 4.80 \\
\hline $\begin{array}{l}\text { CPU-time [sec] } \\
(R([0,5]))\end{array}$ & 0.07 & 0.10 & 0.19 & 0.70 & 2.79 \\
\hline total CPU-time [sec] & 0.11 & 0.19 & 0.41 & 1.85 & 7.59 \\
\hline
\end{tabular}

$$
\begin{aligned}
\hat{A} & =\left[\begin{array}{ccccc}
0.05 & 0.05 & 0 & 0 & 0 \\
0.05 & 0.05 & 0 & 0 & 0 \\
0 & 0 & 0.2 & 0.2 & 0 \\
0 & 0 & 0.2 & 0.2 & 0 \\
0 & 0 & 0 & 0 & 0.2
\end{array}\right] \\
V & =\left[\begin{array}{lllll}
{[0.8,1.2]} & 0 & 0 & 0 & 0
\end{array}\right]^{T}
\end{aligned}
$$

In Fig. 4, one can see projections of the reachable set of the second example. Additionally, the reachable set for higher order systems has been computed which are generated by catenation of instances of the second examples. The computation times are presented in table I. The first row shows the CPU-time for the computation of $\left\lceil e_{p}^{\mathcal{A} r}\right\rceil, F$ and $\bar{R}([0, r])$. The second row presents the CPU-time for the execution of algorithm 1 which returns $R([0,5])$, and the last row lists the total CPU-time. The computations were performed on a dual core notebook processor $(1.66 \mathrm{GHz})$ in Matlab.

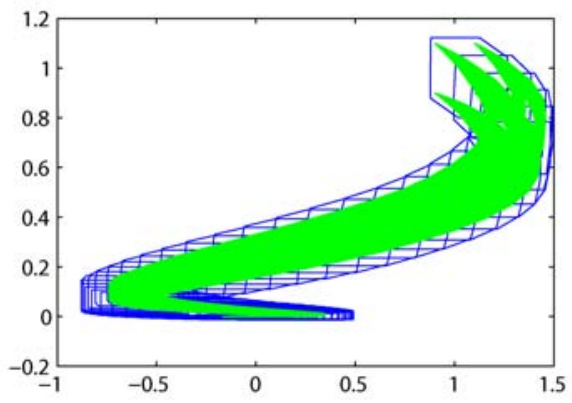

(a) Projection on coordinates $x_{2}$ and $x_{3}$

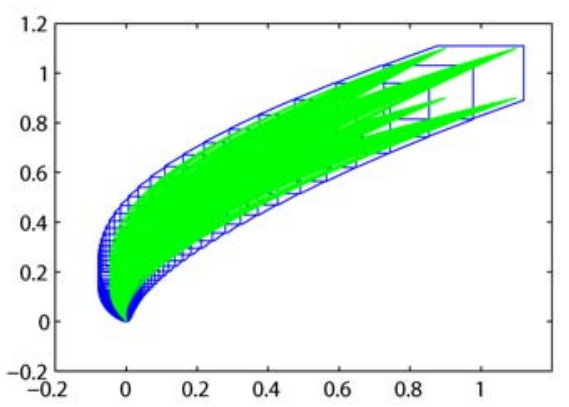

(b) Projection on coordinates $x_{4}$ and $x_{5}$

Fig. 4. Reachable sets for the 5 dimensional example

\section{CONCLUSIONS}

The computation of reachable sets for the class of linear systems with uncertain system matrices and inputs has been presented. Due to the use of zonotopes, the computational complexity grows moderately with dimension $n$ compared to other approaches, such as the computation with arbitrary polytopes. It is noteworthy that the computation of systems with dimension up to 100 can be performed in a few seconds. The presented methods are currently embedded in verification tools for hybrid systems.

\section{ACKNOWLEDGEMENTS}

The authors gratefully acknowledge support of this work by the Deutsche Forschungsgemeinschaft (German Research Foundation) within the Transregional Collaborative Research Centre 28 "Cognitive Automobiles".

\section{REFERENCES}

[1] E. Asarin, T. Dang, G. Frehse, A. Girard, C. Le Guernic, and O. Maler, "Recent progress in continuous and hybrid reachability analysis," in Proc. of the 2006 IEEE Conference on Computer Aided Control Systems Design, 2006, pp. 1582-1587.

[2] G. Lafferiere, G. Pappas, and S. Yovine, "A new class of decidable hybrid systems," in Hybrid Systems: Computation and Control, ser. LNCS 1569. Springer, 1999, pp. 137-151.

[3] T. Henzinger, The theory of hybrid automata, ser. NATO ASI Series F: Computer and Systems Sciences. Springer, 2000, vol. 170, pp. 265-292.

[4] G. Frehse, "Phaver: Algorithmic verification of hybrid systems past hytech." in Hybrid Systems: Computation and Control, 2005, pp. 258273.

[5] A. Chutinan and B. H. Krogh, "Computational techniques for hybrid system verification," in IEEE Transactions on Automatic Control, vol. 48, no. 1,2003 , pp. 64-75.

[6] E. Asarin, O. Bournez, T. Dang, and O. Maler, "Approximate reachability analysis of piecewise-linear dynamical systems," in Hybrid Systems: Computation and Control, 2000, pp. 20-31.

[7] A. Girard, "Reachability of uncertain linear systems using zonotopes," in Hybrid Systems : Computation and Control, vol. 3414, 2005, pp. 291-305.

[8] E. Asarin, T. Dang, and O. Maler, "d/dt: A verification tool for hybrid systems," in Proc. of the Conference on Decision and Control, 2001.

[9] A. B. Kurzhanski and P. Varaiya, "Ellipsoidal techniques for reachability analysis," in Hybrid Systems: Computation and Control, 2000, pp. 202-214.

[10] E. P. Oppenheimer and A. N. Michel, "Application of interval analysis techniques to linear systems: Part i - fundamental results," IEEE Transactions on Circuits and Systems, vol. Volume 35, pp. 1129 1138, 1988.

[11] _ "Application of interval analysis techniques to linear systems: Part ii - the interval matrix exponential function," IEEE Transactions on Circuits and Systems, vol. 35, pp. 1230 - 1242, 1988.

[12] _ - "Application of interval analysis techniques to linear systems: Part iii - initial value problems," IEEE Transactions on Circuits and Systems, vol. 35, pp. 1243 - 1256, 1988.

[13] L. Jaulin, M. Kieffer, and O. Didrit, Applied Interval Analysis. Springer, 2006.

[14] O. Kosheleva, V. Kreinovich, G. Mayer, and H. T. Nguyen, "Computing the cube of an interval matrix is np-hard," in Proc. of the 2005 ACM symposium on Applied computing, 2005, pp. 1449-1453.

[15] C. Moler and C. V. Loan, "Nineteen dubious ways to compute the exponential of a matrix, twenty-five years later," SIAM Review, vol. 45, no. 1, pp. 3-49, 2003.

[16] M. L. Liou, "A novel method of evaluating transient response," in Proceedings of the IEEE, vol. 54, no. 1, 1966, pp. 20-23.

[17] T. Dang, "Vérification et synthèse des systèmes hybrides," Ph.D. dissertation, Institut National Polytechnique de Grenoble, 2000.

[18] O. Stursberg and B. H. Krogh, "Efficient representation and computation of reachable sets for hybrid systems," in Hybrid Systems Computation and Control, ser. LNCS 2623. Springer, 2003, pp. 482497.

[19] J. Zemke, "b4m - A free interval arithmetic toolbox for Matlab based on BIAS," Arbeitsbereich Technische Informatik III 4-04, Technische Universität Hamburg-Harburg, Tech. Rep., 1999. 